UCRL-JC-119287

PREPRINT

\title{
A Low Diffraction Beam Technique for Scanning in the Shallow Ocean
}

\author{
D. K. Lewis
}

This paper was prepared for submittal to the Proceedings of the Full Field Inversion Methods

in Ocean and Seismic Acoustics

A Nato Conference

Lerici, Italy

June 27-July 1, 1994

December 1, 1994

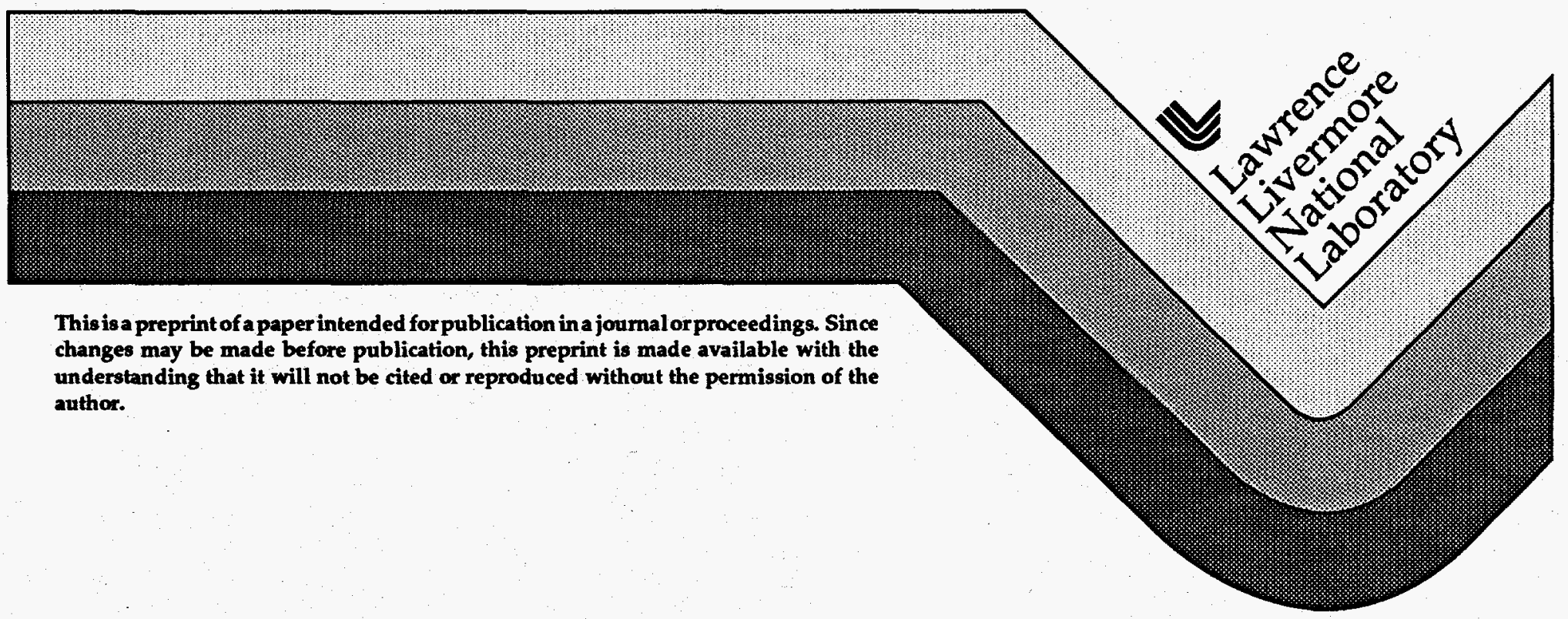




\section{DISCLAIMER}

This document was prepared as an account of work sponsored by an agency of the United States Government. Neither the United States Government nor the University of California nor any of their employees, makes any warranty, express or implied, or assumes any legal liability or responsibility for the accuracy, completeness, or usefulness of any information, apparatus, product, or process disclosed, or represents that its use would not infringe privately owned rights. Reference herein to any specific commercial product, process, or service by trade name, trademark, manufacturer, or otherwise, does not necessarily constitute or imply its endorsement, recommendation, or favoring by the United States Government or the University of California. The views and opinions of authors expressed herein do not necessarily state or reflect those of the United States Government or the University of California, and shall not be used for advertising or product endorsement purposes. 


\section{DISCLAIMER}

Portions of this document may be illegible in electronic image products. Images are produced from the best available original document. 


\title{
A LOW DIFFRACTION BEAM TECHNIQUE FOR SCANNING IN THE SHALLOW OCEAN
}

\author{
D. Kent LEWIS \\ Mail Stop L - 495 \\ Lawrence Livermore National Laboratory \\ Livermore, CA 94550, U.S.A.
}

\begin{abstract}
The Localized Wave pulse project has created the concept of highly localized, pulsed wave energy. We have confirmed, and developed the technique in a series of experimental and numerical tests. This technique generates radiation, mechanical or electromagnetic, from an array. LW generation sends a broad frequency bandwidth pulse of energy along the array axis. The pulse has no sidelobes and maintains the frequency bandwidth and pulse amplitude better than any other method proposed to date. Confirmation of the theory by acoustic experiments show that the effect is real, and that the advantages of the technique can be realized in a laboratory environment. Numerical studies have shown that this effect is extendible to the microwave (vector field) and elastodynamic (tensor field) regimes as well as the stratified ocean environment.

The main features of the LW beam are 1) the pulses are broad bandwidth and maintain that bandwidth over their entire working range; 2) the beam generates no side lobes; 3) the LW effect is accomplished in the linear regime, so that small errors in beam generation result in a less effective beam not the loss of the effect; 4) the beam can be generated using present technology in the acoustic realm.

The LW beam has a wide range of applications. As an active source, it offers a unique method of variable beam forming from spatially wide to the narrowest possible beam. Its uses include oceanographic sound velocity profile estimation and attenuation measurements. Since the pulse characteristics are changeable through the source signal parameters, it also offers the possibility of adaptive beam forming in real time.

Passively, the LW array can use a wide spatial width listening pattern until something of interest occurs. Instantaneously, the listening pattern could be changed to a very narrow, steerable beam with a broad bandwidth. These uses also apply in the electromagnetic realm.

Specific ocean applications include imaging in the shallow ocean, characterization of the ocean bottom and inclusions embedded in it, secure communications and broad bandwidth imaging over long ranges. The lack of side lobes and the wide bandwidth lend the beam to characterization of scattering targets as SONAR cannot do.
\end{abstract}

\section{Introduction}

The Localized Wave (LW) is a pulsed, broad bandwidth beam which maintains its beam width and bandwidth better than any other beams currently available. It is generated without side lobes and depends on individually addressable array technology to put the correct energy at the correct place at the correct time. It uses simple solutions to the wave equation which are superposed to create the desired pulse characteristics. The LW concept is applicable to any linear wave regime, acoustic, electromagnetic or elastodynamic.

The main features of the LW beam are 1) the pulses are broad bandwidth and maintain that bandwidth over their entire range; 2) the beam generates no side lobes; 3) the LW effect is accomplished in the linear regime, so that small errors in beam generation result in a less effective beam not the loss of the effect; 4) the beam can be generated using present technology in the acoustic realm. 
The beam is the result of converging interests. Jim Brittingham at LLNL searched for years to find a transverse solution to Maxwell's equations. When he found it, he termed it the Focus Wave Mode (FWM $)^{(1)}$. The FWM is a perfect waveform since it travels forever without changing its shape or losing its energy. It is also physically impossible in exactly the same way that infinite plane waves are, since it requires an infinite amount of energy and an infinitely large aperture to create.

Rick Ziolkowski, also at LLNL, reasoned that while it is true that both the FWM and plane waves were impossible to create, we use plane waves to simulate wave fields constantly, so if he could find a method of combining the FWM family of basis functions into a physically realizable pulse, he could create a highly localized beam. He searched for a spectrum which would let him do that, and he finally found one that works. He called this spectrum the Modified Pulse Spectrum (MPS) ${ }^{(2)}$.

The resulting source signals are rather simple as illustrated in figure 1. These source functions are for a $1 \mathrm{~cm}$ diameter circular array consisting of a central spot and 5 surrounding rings. The effective frequency of these signals was such that the effective wavelength was approximately equal to the radius of the array.

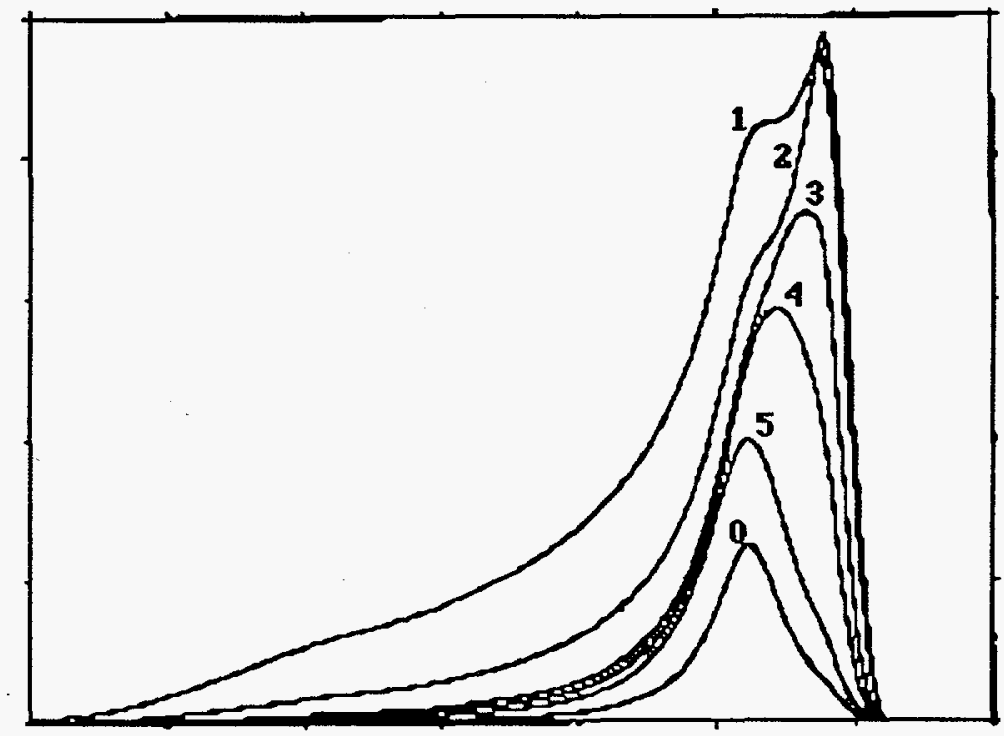

Figure 1. LWT source signals for a 25 element square array. Relative linear amplitudes for central element $(0)$, nearest neighbors (1) etc. Time axis is 7 microseconds.

At the same time I was searching for a broad bandwidth acoustic beam to improve the imaging capabilities of a scanning system. Hearing of the LW beam from a consultant, Bill Cook of the University of Houston, I talked with Ziolkowski about trying to create the pulses in the acoustic domain. This meant rescaling the frequencies into the $\mathrm{MHz}$ range and launching the pulse in water. This was possible since we had a programmable digital to analog converter that worked in this frequency range. This began a period of tightly coupled simulations and experiments which were to confirm the feasibility of the LW pulse $(3,4,5,6)$. 


\section{Signal Preprocessing}

The pulse was subjected to both numerical and experimental testing. Numerically, we examined the predictions of simulations in both time and frequency based models. In the time domain we used both point to point phasing and ray tracing techniques. Frequency simulations launched the different frequencies of the source array and recombined them to create the pulse. All of these methods produced the same results, and these results agreed with the theoretical predictions.

One aspect of the LW project was the use of array folding and time gating. Array folding assumes that the aperture is larger than the actual aperture. By using a single folding point on the axis as a reference, each signal from the virtual part of the array is mapped into a part of the existing array. Using this technique, there is only one point, the folding point, at which the actual field is that which would have been created by the larger array. But near this point the field is approximately the same.

A measure of the accuracy of the field can be represented by what we can imagine as a similarity parameter. Obviously at the array surface, the field created is infinitely different than that of the virtual array. Similarly as the field approaches infinity the field will be different since the signals launched are different than the virtual signals. But over the range of the beam, the agreement is sufficiently good to increase the localization of the pulse.

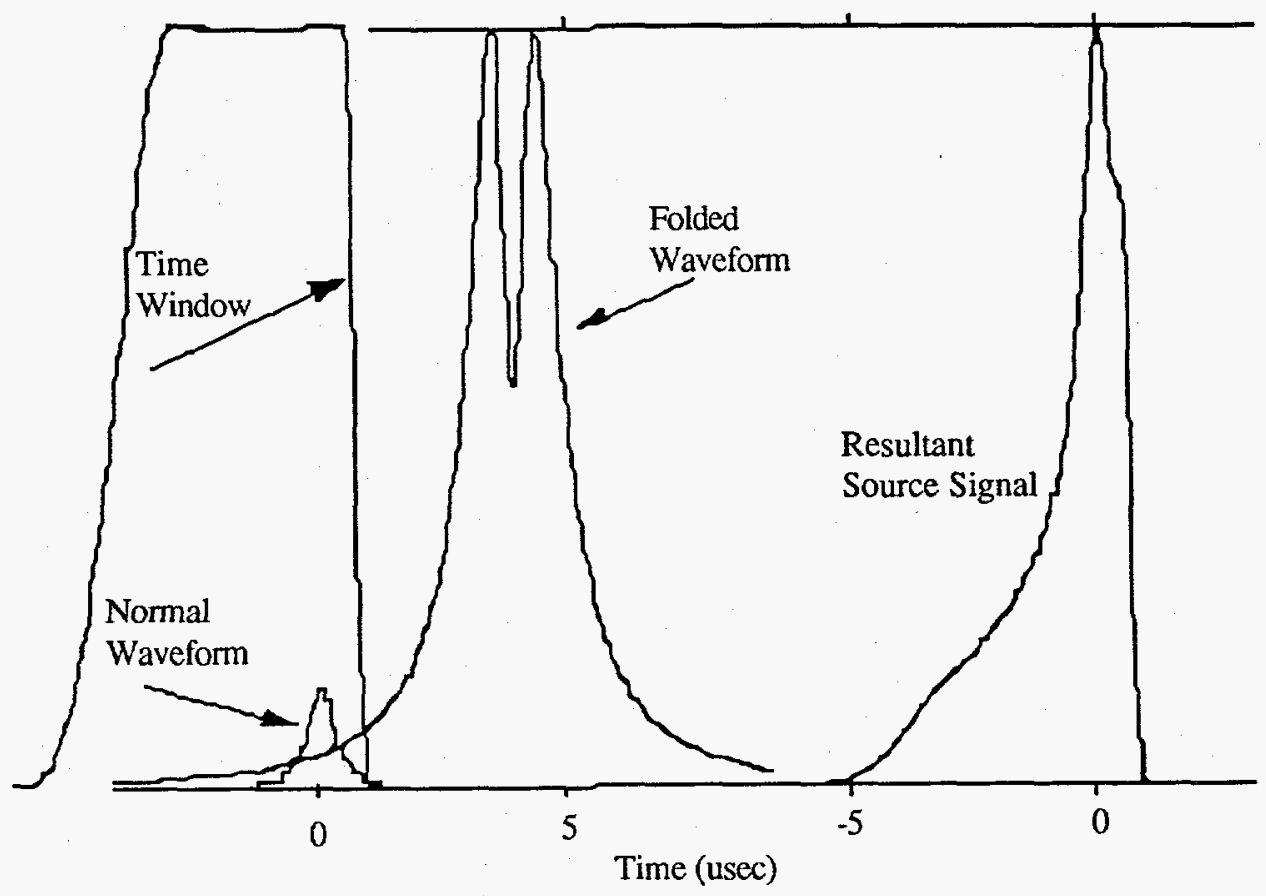

Figure 2. Formation of the folded signal for an array element. Normal Waveform is the signal prescribed by theory fo rhte element, Folded Waveform is the signal from a virtual element time shifted to arrive at the folding point at the proper time and magnified by the ratio of virtual element area to actual area. The signals are summed and windowed as shown to arrive at the Resultant Waveform.

Time windowing is a treatment of the combined real element and virtual element signals which increases the efficiency of the beam. When we used the folding technique, we enhanced the 
localization of the pulse dramatically, but we noticed that there was now more energy in the off axis space of the pulse. This energy was from that part of the folded signals which were not needed for the selected range of the particular designed beam. In order to increase the efficiency without decreasing the localization, we experimented with different time windowing schemes.

As shown in figure 2, the small, original signal and the larger, folded signal, are modified by another signal which throws away most of the folded signal. The resulting signal, different from both the original signals, is shown also with a normalized amplitude. These signals, like those of the first illustration, are still very simple signals, and very easy to generate.

\section{Experimental Tests}

The experimental methods included acousto-optical field measurements, two transducer simulation measurements, and sound field measurements from a 5\% active area array and an $80 \%$ active area array. The first two experiments were simulated array measurements in that the source signals were generated separately and combined in a computer. The last two experiments were of active arrays actually launching the LW pulse.

During the development of this beam, we have developed the technologies of individually addressable acoustic arrays and of source signal pre-processing. Our multi-channel electronics are applicable to existing acoustic array equipment and lend them selves to massively parallel computer interfaces.

The pre-processing technique uses sampling of the source signals and inverse filter processing to tailor the source signals for the optimum element response. This allows us to launch as many individual signals as necessary to our array elements and to overcome element to element feedback interactions. Preliminary discussions with vendors encourages us to believe that present ocean arrays may be of sufficient bandwidth to be used in further development.

The experimental tests of the $\mathrm{LW}$ beam have involved several different techniques. In the first set of tests, to determine if the source signals could be accurately launched, an acousto-optic technique was used. In this technique a laser beam is used to probe the sound field so that a measurement can be made without changing the field. Predicted and measured fields were extraordinarily similar.

Since the acousto-optic technique is actually measurement of the change to a light beam over a distance caused by the sound field, the model of this experiment was of an array of line sources radiating to a point receiver. The confirmation of the theory in these tests, 1 dimensional array, led us to try the next logical step of a circular array.

The simulated 2 dimensional array results were also positive, so preparations were made to design and test a real array. The first array tested, with all the source signals launched at the same time, was a 25 element array consisting of $1.2 \mathrm{~mm}$ current feeds at $2.5 \mathrm{~mm}$ center to center distances. The elements were fed signals to simulate a central spot and 5 surrounding rings. The active piezoelectric was a PVDF sheet with a ground plane on the outer face. The active area was only $5 \%$ of the total array size.

In this series of tests, the beam achieved better than 10 times the near field distance compared to a conventional beam of the same effective frequency. We then constructed an array of a central spot and 10 surrounding rings. This array had an active area which was $80 \%$ of the total array surface. This beam did not perform as well as the previous one, and achieved only a factor of slightly greater than 3 times the near field expected. We believe that much of the energy went into deforming the PVDF co-polymer between the very close rings. 
The results of the latest test were clearly better than expected as seen in the final figure. Figure 3 shows the fields from the $2 \mathrm{~cm}$ array at a distance of $100 \mathrm{~cm}$ downrange for the $L W$ pulse at an effective frequency of $205 \mathrm{kHz}$, a $250 \mathrm{kHz}, 500 \mathrm{kHz}, 1 \mathrm{MHz}$ and $2 \mathrm{Mhz}$ tone bursts. It should be noted that the LW beam achieved nearly the localization of the $2 \mathrm{MHz}$ beam without any side lobes, with a broad bandwidth, and with smooth behavior close to the array in contrast to the complicated behavior of a single frequency toneburst.

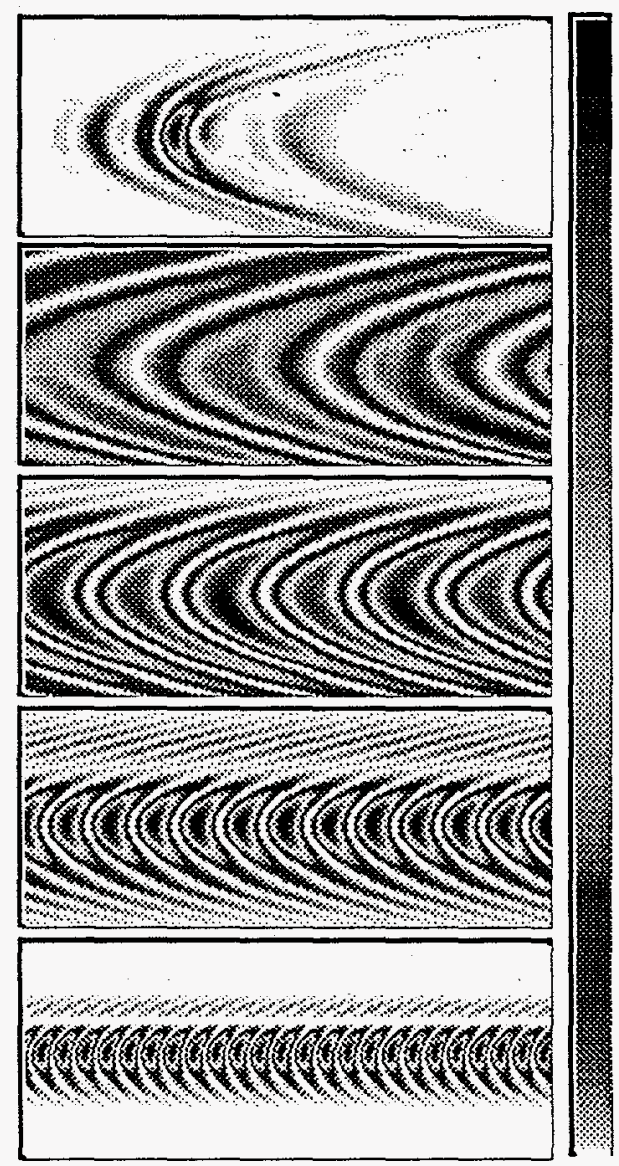

Figure 3. Linear intensity fields of $\mathrm{LW}$ pulse (top), $250 \mathrm{kHz}, 500 \mathrm{kHz}, 1 \mathrm{MHz}$ and $2 \mathrm{MHz}$ tonebursts $100 \mathrm{~cm}$ downrange from a $2 \mathrm{~cm}$ diameter array. The field widths are $6 \mathrm{~cm}$ and the effective frequency of the $\mathrm{LW}$ pulse is $205 \mathrm{kHz}$. Intensity color map is at right.

\section{Applications}

The LW beam is immediately applicable for shallow water conditions. It is useful for identifying and locating floating, submerged and buried mines, scanning for and identification of submarines and characterization of ocean parameters such as the SVP, attenuation caused by reverberation, sediment and ocean floor wave speeds. Since it is a narrow pulse with no side lobes, it is possible to avoid many of the interactions that plague SONAR arrays.

In Biot solids, such as saturated sand or sediment, the beam offers a tight profile which overcomes the limitations of single frequency beams. A relatively high frequency beam suffers scattering loss since the particles are large compared to the wavelength. A tight focus is 
maintained, but insufficient power returns for effective scanning. A low frequency beam overcomes the scattering limits but loses power through beam spreading since the wavelength is large relative to the array size. The LW beam is a tight beam pattern caused by superposition of low frequency waves, so if the beam behaves as one would expect, it can return more power in a tighter beam.

For passive detection using an array, the techniques of the LW pulse, specific frequency filtering for each element, can be used for improved beam forming. The same lack of side lobes and higher localization over the effective range can be brought to passive listening techniques. Combined with the beam generator, there is the possibility of better than inverse fourth power scanning.

The LW beam is also useful for nondestructive evaluation applications such as inspection of composite structures. Carbon composites or metal matrix composites offer the same problems as a Biot solid and the high frequency behavior of the low frequency LW pulse offers better penetration, broader bandwidth and higher return field for these difficult to inspect materials. The passive technique is also possible with present day technology.

\section{References}

1. “J. B. Brittingham, J. Appl. Phys. 54, 1179 (1983)

2. "R.W.Ziolkowski, J. Math. Phys. 26, 861 (1985)

3. "R.W.Ziolkowski, D. K. Lewis and B.D.Cook, Phys. Rev. Let. 62, 147 (1989)

4. "J. V. Candy, D. K. Lewis and R. W. Ziolkowski, J. Acous. Soc. of Am. 88, 2235 (1990)

5. “J. V. Candy, D. K. Lewis and R. W. Ziolkowski, J. Acous. Soc. of Am. 88, 2248 (1990)

6. R. W. Ziolkowski and D. K. Lewis, J. Appl. Phys. 68, 6083 (1990)

\section{Acknowledgement}

This work was performed in part by the Lawrence Livermore National Laboratory for the U. S. Department of Energy under Contract No. W-7405-ENG-48. 